\title{
Management of pulmonary metastases: the surgeon's perspective
}

\author{
Piergiorgio Muriana $^{1} \wedge$, Giovanni Muriana² \\ ${ }^{1}$ Department of Thoracic Surgery, IRCCS San Raffaele Scientific Institute, Milan, Italy; ${ }^{2}$ Italian Society of Thoracic Surgery, Rome, Italy \\ Correspondence to: Piergiorgio Muriana, MD. Department of Thoracic Surgery, IRCCS San Raffaele Scientific Institute, Via Olgettina 60 - 20132 \\ Milan, Italy. Email: muriana.piergiorgio@hsr.it.
}

Received: 24 May 2021; Accepted: 21 June 2021; Published: 30 December 2021.

doi: $10.21037 /$ asj-21-33

View this article at: https://dx.doi.org/10.21037/asj-21-33

Lungs are among the most frequent targets of hematogenous metastases from solid tumors. Despite the recent advances of oncology in the comprehension of the biological mechanisms of carcinogenesis and the introduction of new targeted therapies, a considerable number of patients affected by solid tumors still develops pulmonary metastases in their clinical course. The number of undetermined pulmonary nodules encountered on CT-scan of oncological patients is very high. In a study by Hanamiya et al., one or more lung incidentalomas were found in $75 \%$ of cases in 308 patients during staging CT-scan (1). In another trial by Caparica and colleagues including patients with extrapulmonary malignancies undergoing biopsy for the differential diagnosis of undetermined lung nodules, over $60 \%$ showed the presence of metastatic disease (2). The incidence of pulmonary metastases development has been reported to be the highest in patients affected by bone and soft tissue sarcomas, melanomas, germ cell tumors, and kidney tumors; nevertheless, almost all types of cancer may potentially spread to lungs in the clinical course of the disease (3).

Surgery has historically played a key role in the treatment of pulmonary metastatic disease. The first report of surgical treatment of pulmonary metastases dates back to $19^{\text {th }}$ century. In 1971, Martini et al. showed improved long-term survival in a series of 22 patients surgically treated for pulmonary metastases from osteogenic sarcoma; several of these patients underwent multiple thoracotomies, introducing the concept of iterative surgery for the local control of metastatic disease (4). A recent meta-analysis of 9 studies and 464 patients confirmed the results of the group of Martini, showing a $50 \%$ reduction in the risk of death following repeated surgical excision of lung metastasis from sarcoma (5). In the last decades, plenty of studies have demonstrated that surgery represents a valid option in the multimodal treatment of patients affected by pulmonary secondary cancers.

Certainly, a number of factors strongly influence survival after pulmonary metastasectomy, such as the site of primary tumor. In fact, while surgical excision of lung metastasis can significantly improve survival in patients with colorectal and renal cell cancers, the role of surgery is still under discussion in case of breast cancer (6-9). Moreover, other aspects (disease-free interval before of the onset of metastatic disease, number and diameter of lesions, and the involvement of loco-regional lymph nodes) additionally greatly affect long-term results of the surgical treatment.

By the time of publication of the first series of pulmonary metastasectomies, surgeons worldwide followed few basic principles to decide patient eligibility for treatment: first, the primary tumor has to be treatable and no extrapulmonary metastatic disease should be present at the time of surgery; second, there should be no functional contraindications for lung resection; and no better alternative therapy should be available (10).

Despite considerable long-term survival has been reported for patients affected by several tumor histotypes, the real value of the surgical treatment in the field has been questioned. In the paper by Treasure et al., it was pointed out that the outcomes of the surgical treatment of secondary lung cancers essentially rely on conclusions drawn from retrospective clinical series, and the results of

^ ORCID: 0000-0003-1341-1071. 
these studies are deeply biased by preoperative selection of patients with positive prognostic factors (11). According to the authors, randomized controlled trials (RCTs) and comparative studies are therefore advocated to estimate the current role of pulmonary metastasectomy. To date, the only available RCT is the Pulmonary Metastasectomy in Colorectal Cancer (PulMiCC Trial) (12). Forty-six patients underwent surgical resection of lung metastases from primary colorectal cancer and were compared to 47 patients who did not receive local treatments [surgery or stereotactic body radiotherapy (SBRT)]. The study, however, showed comparable median survival (3.5 vs. 3.8 years respectively) and 5-year risk of death between the groups (hazard ratio $=0.93)$. Moreover, this RCT was closed early ahead of randomization of 300 planned cases due to difficulties encountered in the enrollment of patients, a problem that has previously limited the feasibility of comparative studies in metastatic patients.

Thus, the role of surgery for the treatment of pulmonary metastases from solid tumors is nowadays still controversial, with a number of questions to be solved: may minimally invasive video-assisted thoracic surgery (VATS) represent the optimal surgical approach ensuring both appropriate evaluation of lung parenchyma in the search of metastases undetected by preoperative imaging studies and fast postoperative recovery for subsequent systemic therapies? Is systematic lymph node dissection always required when performing lung metastasectomy, or should it be offered in selected cases only? Which patients can benefit of iterative surgical excision of secondary lung localizations and what is the expected survival benefit (13-16)?

While waiting for new RCTs to be designed, a significant role can be covered by clinical registries. In fact, in a heterogenous field like that of the surgical treatment of pulmonary metastases, clinical registries may have the advantage of providing data reflecting the so-called 'realworld' experience rather than that inferred from the experimental environment of RCTs (17). An effective clinical registry, following the principles identified by Klaiman et al. (data standardization, transparency, accuracy and completeness of data, participation by providers, financial sustainability, feedback to providers), allows researchers to obtain continuative information from nonhomogenous patient populations along with a strong external validation for scientific purposes (18).

The International Registry of Lung Metastases (IRLM) was instituted in 1990 by several European and NorthAmerican centers with the aim of overcoming controversies on the selection of patients, indications to surgical treatment, and appropriateness of postoperative adjuvant therapy, and to possibly identify reproducible clinical and pathological prognostic factors in the field of secondary lung cancers. In 1997, the outcomes of a cohort of over 5,000 patients included in the database were presented by Pastorino (19). In this study, a high rate of long-term survival was observed in patients with resectable disease, in those with single lung metastasis and in patients with a disease-free interval (DFI) between primary tumor diagnosis and lung metastasectomy longer than 36 months. These factors were further used to classify patients in four prognostic categories: (I) no risk factors; (II) resectable disease +1 risk factor between DFI ( $<36$ months) and number of lesions $(\geq 2)$; (III) resectable disease +2 risk factors; and (IV) unresectable disease. By that time, the authors concluded that pulmonary metastases from solid tumors are treatable and amenable of long postoperative survival, also considering the possibility of iterative interventions; nevertheless, non-invasive radiological investigations and VATS showed inadequate sensibility in the detection of lung lesions compared to manual palpation in an era prior to the definitive development of nuclear medicine technology. The major limitations of the IRLM were the inability to assess the prognostic role of factors related to specific tumors and that of salvage surgery across different histologies.

In 2006, the European Society of Thoracic Surgeons (ESTS) endorsed the formation of a working group for the development of specific guidelines on the diagnostic and surgical management of pulmonary metastases. However, a survey launched by the working group among the members of the Society (both European and extra-European), despite showing a common agreement on the indications to metastasectomy (primary tumor control and lung lesions resectability), reported great heterogeneity in particular when considering preoperative imaging and the optimal surgical approach, not deviating far from the experience of the IRLM (20). Therefore, in the light of the results of this survey and of a series of papers published in a dedicated issue of the Fournal of Thoracic Oncology in 2010, the working group came to the conclusion that it is not yet possible to establish definitive guidelines for the management of pulmonary metastases with a strong level of evidence (21).

Based on these controversial factors, in 2009 we founded the Italian Clinical Registry of Pulmonary Metastases (REgistro Clinico Italiano delle Metastasi Polmonari RECIMP) under the endorsement of the Italian Society of 


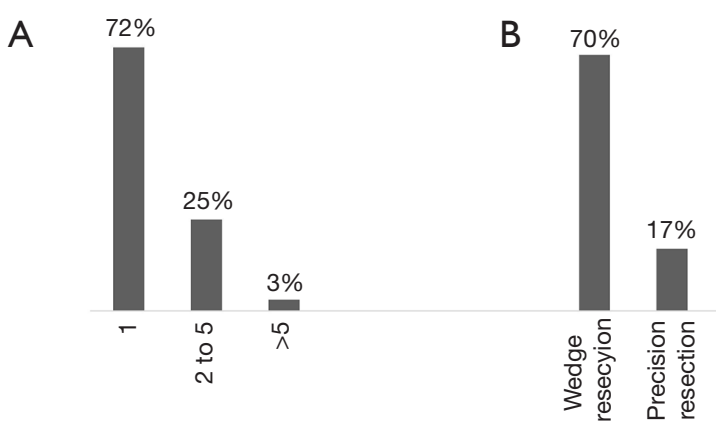

D

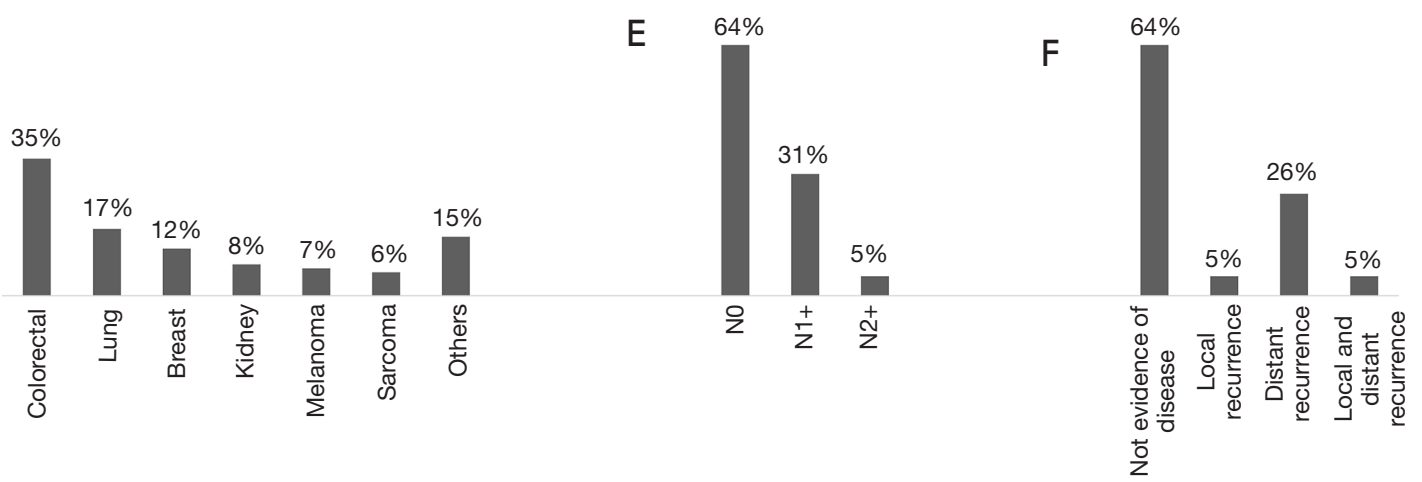

Figure 1 Provisional data of patients enrolled in the RECIMP. (A) Number of resected metastases; (B) type of pulmonary resection; (C) surgical approach; (D) final pathologic results of resected lesions; (E) pathologic lymph nodal status; (F) postoperative follow-up. VATS, video-assisted thoracic surgery; RECIMP, the Italian Clinical Registry of Pulmonary Metastases (REgistro Clinico Italiano delle Metastasi Polmonari) .

Thoracic Surgery (SICT). Since September 2010, patients undergoing surgical resection with radical intent of lung metastases from solid primary tumors were prospectively enrolled in the registry by 40 joining centers in Italy.

The establishment of this registry was important for several reasons. First, considering the variability in the management of pulmonary metastases, it could give us an insight into the actual practice at a national level in the attempt to evaluate results in a homogenous population. It should be anyway noted that Italian centers covered an important role both in IRLM and ESTS experiences. Second, the registry was deliberately designed on a multidisciplinary basis to integrate surgical data with those regarding oncological chemo- and radiotherapy treatments. In fact, in the modern era of thoracic oncology, we firmly believe that therapeutic planning ought to be jointly agreed by both surgical and medical component of the multidisciplinary disease team, both at preoperative and postoperative levels, as recently strongly recommended by the Workforce on Evidence Based Surgery of the Society of Thoracic Surgeons (STS) (22). Finally, although not directly connected to lung metastases treatment, the established of RECIMP constituted an important experience for the later development of the Italian Registry of VATS lobectomy (VATS-group), that is currently an important tool to improve the technical and clinical knowledge on the minimally invasive treatment of lung cancer in Italy, based on data of over 12,000 patients (23).

Thus far, almost 1,900 patients data have been entered in the RECIMP and 3 research protocols based on the registry are currently under evaluation by the SICT. Provisional results are showed in Figure 1. Despite the enrollment of patients is still ongoing, some interesting data could be obtained processing the currently available information.

As expected, about three-quarters of cases were treated for a single metastasis, while resection of multiple lesions (more than five) was documented only in $3 \%$ of cases. In $70 \%$ of patients the target lesions resulted resectable in the 
volume of an atypical wedge resection, a rate increasing up to $90 \%$ if considering all types of sublobar pulmonary resections. Pneumonectomy was required only in very few selected cases-1\% compared to $3 \%$ of the IRLM (19). In fact, a subgroup analysis of the latter registry showed that patients who underwent pneumonectomy for metastases treatment had a 5-year survival of $20 \%$, with an intraoperative mortality of $4 \%$, increasing up to $19 \%$ in case of non-radical resection (24). Hence, the growing tendency is to preferentially avoid surgery in patients potentially candidates to pneumonectomy. Only $5 \%$ of patients in the RECIMP had incomplete resections, and less than $10 \%$ of patients underwent adjuvant postoperative chemo- and/or radiotherapy. Colorectal, breast, and lung cancer were the most common primary histotypes involved.

The therapeutic and prognostic role of lymph node dissection in the course of pulmonary metastasectomy is still unclear. Indeed, the Consensus Document released by STS reports low agreement between responders when considering the necessity of nodal assessment in patients operated for lung metastases (22). Still, in 36\% of patients from RECIMP N1 or N2 positive lymph nodes were found at final pathologic examination. This finding should be further analyzed in future because of its potential prognostic implications.

Despite the recent advancements of VATS in Italy, an especially significant result from our registry is that the preferred surgical approach was still open thoracotomy in over $50 \%$ of cases overall. On the other side, techniques which may allow exploration of both lungs, such as median sternotomy and clamshell incision/bilateral thoracotomy, were reported in few cases. This outcome probably reflects the diffuse controversial need to confirm preoperative noninvasive findings by intraoperative manual palpation.

At follow-up, two-thirds of all the patients enrolled are currently alive, $64 \%$ of which with no evidence of disease.

Certainly, the results of RECIMP are still far from being conclusive at their present stage, and closely reflect the experience of previous registries. Many are the challenges of the near future to define the optimal treatment of pulmonary metastases. Alternative treatments, such as SBRT and targeted therapies have emerged in recent years with very promising short-term outcomes, but yet uncertain long-term results compared with surgery (25). Hopefully, the role of clinical registries-and possibly of forthcoming RCTs - will be that of creating evidence-based guidelines shared on a multidisciplinary level, including all therapeutic options currently available and those that will be furtherly introduced in our practice.

\section{Acknowledgments}

The authors thank Dr. Francesca Rossetti for English language review.

Funding: None.

\section{Footnote}

Provenance and Peer Review: This article was commissioned by the Guest Editors (Davide Tosi, Alessandro Palleschi and Paolo Mendogni) for the series "Management and Treatment of Lung Metastases" published in AME Surgical fournal. The article did not undergo external peer review.

Conflicts of Interest: Both authors have completed the ICMJE uniform disclosure form (available at https://asj.amegroups. com/article/view/10.21037/asj-21-33/coif). The series "Management and Treatment of Lung Metastases" was commissioned by the editorial office without any funding or sponsorship. The authors have no other conflicts of interest to declare.

Ethical Statement: The authors are accountable for all aspects of the work in ensuring that questions related to the accuracy or integrity of any part of the work are appropriately investigated and resolved.

Open Access Statement: This is an Open Access article distributed in accordance with the Creative Commons Attribution-NonCommercial-NoDerivs 4.0 International License (CC BY-NC-ND 4.0), which permits the noncommercial replication and distribution of the article with the strict proviso that no changes or edits are made and the original work is properly cited (including links to both the formal publication through the relevant DOI and the license). See: https://creativecommons.org/licenses/by-nc-nd/4.0/.

\section{References}

1. Hanamiya M, Aoki T, Yamashita $Y$, et al. Frequency and significance of pulmonary nodules on thin-section CT in patients with extrapulmonary malignant neoplasms. Eur J Radiol 2012;81:152-7.

2. Caparica R, Mak MP, Rocha CH, et al. Pulmonary Nodules in Patients With Nonpulmonary Cancer: Not Always Metastases. J Glob Oncol 2016;2:138-44.

3. Hayman J, Naidoo J, Ettinger DS. Lung Metastases. In: Niederhuber JE, Armitage JO, Doroshow JH, Tepper JE, 
editors. Abeloff's Clinical Oncology, 6th ed. Philadelphia: Elsevier; 2020. p. 831-46.

4. Martini N, Huvos AG, Miké V, et al. Multiple Pulmonary Resections in the Treatment of Osteogenic Sarcoma. Ann Thorac Surg 1971;12:271-80.

5. Li Y, Zhang W, Li S, et al. Clinical efficiency of repeated pulmonary metastasectomy in sarcoma patients with recurrent pulmonary metastasis: A meta-analysis. J Cancer Res Ther 2018;14:S457-62.

6. Salah S, Watanabe K, Park JS, et al. Repeated resection of colorectal cancer pulmonary oligometastases: Pooled analysis and prognostic assessment. Ann Surg Oncol 2013;20:1955-61.

7. Zabaleta J, Iida T, Falcoz PE, et al. Individual data meta-analysis for the study of survival after pulmonary metastasectomy in colorectal cancer patients: A history of resected liver metastases worsens the prognosis. Eur J Surg Oncol 2018;44:1006-12.

8. Zhao Y, Li J, Li C, et al. Prognostic factors for overall survival after lung metastasectomy in renal cell cancer patients: A systematic review and meta-analysis. Int J Surg 2017;41:70-7.

9. Endoh M, Shiono S, Yamauchi Y, et al. Pulmonary metastasectomy for pulmonary metastasis of breast cancer has a limited prognostic impact: a multi-institutional retrospective analysis. J Thorac Dis 2020;12:6552-62.

10. Davidson RS, Nwogu CE, Brentjens MJ, et al. The surgical management of pulmonary metastasis: Current concepts. Surg Oncol 2001;10:35-42.

11. Treasure T, Miloševíc M, Fiorentino F, et al. Pulmonary metastasectomy: What is the practice and where is the evidence for effectiveness? Thorax 2014;69:946-9.

12. Milosevic M, Edwards J, Tsang D, et al. Pulmonary Metastasectomy in Colorectal Cancer: updated analysis of 93 randomized patients - control survival is much better than previously assumed. Colorectal Dis 2020;22:1314-24.

13. Meng D, Fu L, Wang L, et al. Video-assisted thoracoscopic surgery versus open thoracotomy in pulmonary metastasectomy: A meta-analysis of observational studies.

doi: $10.21037 /$ asj-21-33

Cite this article as: Muriana P, Muriana G. Management of pulmonary metastases: the surgeon's perspective. AME Surg J 2021;1:21.
Interact Cardiovasc Thorac Surg 2016;22:200-6.

14. Welter S, Gupta V, Kyritsis I. Lymphadenectomy in pulmonary metastasectomy. J Thorac Dis 2021;13:2611-7.

15. Kim HK, Lee K, Han KN, et al. The predictive accuracy of sentinel nodes mapping in the setting of pulmonary metastasectomy. Clin Exp Metastasis 2017;34:125-31.

16. De Raffele E, Mirarchi M, Casadei R, et al. Twenty-year survival after iterative surgery for metastatic renal cell carcinoma: A case report and review of literature. World J Clin Cases 2020;8:4450-65.

17. Sherman RE, Anderson SA, Dal Pan GJ, et al. Real-World Evidence - What Is It and What Can It Tell Us? N Engl J Med 2016;375:2293-7.

18. Klaiman T, Pracilio V, Kimberly L, et al. Leveraging effective clinical registries to advance medical care quality and transparency. Popul Health Manag 2014;17:127-33.

19. Pastorino U, Buyse M, Friedel G, et al. Long-term results of lung metastasectomy: Prognostic analyses based on 5206 cases. J Thorac Cardiovasc Surg 1997;113:37-49.

20. Internullo E, Cassivi SD, van Raemdonck D, et al. Pulmonary metastasectomy: A survey of current practice amongst members of the european society of thoracic surgeons. J Thorac Oncol 2008;3:1257-66.

21. Van Raemdonck D, Friedel G. The European society of thoracic surgeons lung metastasectomy project. J Thorac Oncol 2010;5:S127-9.

22. Handy JR, Bremner RM, Crocenzi TS, et al. Expert Consensus Document on Pulmonary Metastasectomy. Ann Thorac Surg 2019;107:631-49.

23. Nosotti M, Droghetti A, Luzzi L, et al. First Italian Consensus Conference on VATS lobectomy for NSCLC. Tumori 2017;103:124-35.

24. Koong HN, Pastorino U, Ginsberg RJ. Is there a role for pneumonectomy in pulmonary metastases? Ann Thorac Surg 1999;68:2039-43.

25. Londero F, Grossi W, Morelli A, et al. Surgery versus stereotactic radiotherapy for treatment of pulmonary metastases. A systematic review of literature. Future Sci OA 2020;6:FSO471. 